\title{
Evaluation of balance function in patients with radiologically (CT scan) confirmed otosclerosis
}

\author{
Rania Abdulfattah Sharaf ${ }^{1, *}$, Rudrapathy Palaniappan ${ }^{2}$
}

${ }^{1}$ National Guard health affairs, Ministry of Nation guard, Saudi Arabia

${ }^{2}$ Royal National Throat Nose and Ear Hospital, UCLH NHS Foundation Trust, London, UK

\section{Correspondence}

Rania Abdulfattah Sharaf, National Guard health affairs, Ministry of Nation guard, Saudi Arabia

Email: rania2222@hotmail.com

History

- Received: Jan 02, 2019

- Accepted: Mar 10, 2019

- Published: Mar 29, 2019

DOI : 10.15419/bmrat.v6i3.526

\section{Check for updates}

\section{Copyright}

( ) Biomedpress. This is an openaccess article distributed under the terms of the Creative Commons Attribution 4.0 International license.

\begin{abstract}
Objective: To assess balance function in patients with radiologically confirmed otosclerosis. Methods: Sixteen patients (14 females and 2 males), who attended the Neuro-Otology clinic/ ENT clinics at the Royal National Throat Nose and Ear Hospital, participated in this study. After general medical, audiological and Neuro-Otological examination, patients underwent the caloric and rotational testing. Results: Thirteen of the 16 patients had radiologically confirmed otosclerosis ( 12 females and 1 male). A total of 3 patients (2 females and 1 male) did not have CT confirmation of otosclerosis, and therefore, were excluded from the study. The remaining 13 patients' data were analyzed. Nine patients had a mixed hearing impairment at least on one side, while eight patients had a bilateral mixed hearing loss and one patient had a sensorineural hearing loss on one side. Four patients had a bilateral sensorineural hearing loss. Only 1 patient had a canal paresis (CP) at $35 \%$. None of the patients had any significant directional preponderance (DP). The patient with significant CP (35\%) did not show any rotational asymmetry on impulsive rotation. Eleven patients had a rotational chair test. Only one patient had a significant asymmetry to the right at $25.30 \%$ (normal range is $<20 \%$ ). Overall, $18 \%(n=2)$ of the radiologically confirmed otosclerosis patients showed an abnormal balance test, including both caloric and rotational tests. More than $80 \%(n=9)$ of the patients with radiological otosclerosis showed balance symptoms. Conclusions: the current study indicates a high prevalence of balance symptoms ( $n=9$ or $82 \%$ ) in patients with radiological otosclerosis, although only a small proportion $(n=2$ or $18 \%)$ showed an abnormal vestibular function on caloric and impulse rotation testing. However, due to a small number of samples, further validation of this result is warranted.
\end{abstract}

Key words: Balance, Otosclerosis, Canal paresis, Vestibular function, Hearing impairment

\section{INTRODUCTION}

Balance function studies in otosclerosis were carried out mainly in the early and late 70s, when the diagnosis of this condition was based essentially on audiometric findings without any radiological confirmation. It is, therefore, possible that the incidence of balance disorder reported in otosclerosis may not be a true reflection of the problem. We conducted this study to assess balance function in patients with radiologically (CT scan) confirmed otosclerosis, including both patients with fenestral and cochlear diseases. In addition to typical conductive hearing loss, patients suffering from otosclerosis often have a perceptive hearing loss. Cochlear lesions are caused by the secretions from the active otosclerotic foci, which then enter the endolymph ${ }^{1}$. Vestibular disturbances also appear in patients suffering from otosclerosis ${ }^{2}$, which may be derived from otosclerotic vascular changes due to the disease or from biochemical changes in the inner ear fluids. Because hearing loss is the dominant feature of otosclerosis, these patients may not be examined for other disorders associated with otosclero- sis. Unsteadiness and dizziness have been frequently observed in these patients. Abnormal electronystagmographic (ENG) findings have been reported in otosclerotics $^{3}$. In the last few years, the study of otosclerosis has principally been devoted to the histochemistry of otosclerotic foci ${ }^{4,5}$.

It is important to distinguish between clinical, nonclinical or histological otosclerosis; the latter is about 10 times more common than the former ${ }^{6}$. A study has revealed histological otosclerosis on temporal bones in $12 \%$ of patients ${ }^{7}$. In 1944, Guild demonstrated histological otosclerosis in only $8.3 \%$ of 482 African races, which was much less in the wider population (around 1\%) ${ }^{8}$.

Otosclerosis is usually bilateral; however, the frequency of histological unilateral otosclerosis ranges between $13 \%$ and $30 \%^{8-10}$. The area of the labyrinthine capsule immediately in front of the footplate of the stapes, which is called 'fissula antefenestrum, is the site of predilection for otosclerosis. Other frequent sites may include a border of the round window and even the footplate of the stapes around 
the cochlea, the internal auditory canal, or less often, around the semicircular canal ${ }^{11}$.

Previous studies reported vestibular symptoms in patients with otosclerosis ${ }^{12,13}$. Clinical studies reported the prevalence of vestibular symptoms in 3-35\% of patients with otosclerosis ${ }^{14}$. Another study reported increased caloric abnormalities in ears with cochlear pathology when compared to pure conductive hearing loss ${ }^{15}$. The current study aimed to evaluate balance function in patients with CT scan confirmed otosclerosis.

\section{METHODS}

\section{Subjects}

The total of 16 patients, aged 13-82 (mean age $=56.5)$ and suffering from clinical o tosclerosis, participated in this study. Patients with otosclerosis were recruited from the Neuro-Otology and the ENT clinics at the Royal National Throat N ose \& e ar Hospital (RNT$\mathrm{NEH}$ ). Patients' details of the above two clinics were collected from the respective consultants and they were invited to take part in the study. Patients with radiologically confirmed o tosclerosis were included. Patients with a history of perforated eardrum or ear discharge, ear operations, head injuries, meningitis, and ototoxic medication were excluded. As we already had a normative data for the balance tests from the Neuro-Otology clinic at RNTNEH, the ethics committee did not give permission for caloric testing of normal individuals. Consequently, this study could not have a control group of normal individuals. However, we were able to compare the data from the study with normative data available in the neuro-otology clinic. This study was approved by the Royal Free Hospital and Medical School Local Research Ethics Committee.

After necessary information, instruction and consent, patients had the following tests:

1-Pure tone Audiometry (PTA)

2-Impedance Audiometry (Tympanogram)

3-Bithermal Water Caloric Test

4-Rotational chair Test (ENG)

All patients had the impedance audiometry, pure tone, air, and bone conduction thresholds as per British Society of Audiology (BSA) recommended procedures. All eligible patients had bithermal caloric and rotational chair testing and the response was recorded by electronystagmography. All patients filled in a questionnaire, which included 8 different types of questions regarding to their balance (See $\boldsymbol{a p}$ pendix A). Each participant was requested to provide a written informed consent form before participation.

\section{Physical examination}

Within the clinic, a brief interview was carried out to conform to our inclusion and exclusion criteria. In this initial interview, each patient was asked to fill in the questionnaire and sign the consent form. The outer ear and the tympanic membrane (TM) were examined using a halogen otoscope to exclude any perforations or abnormal looking ear drums prior to the test.

\section{Pure Tone Audiometry (PTA)}

PTA was carried out using a GSI6/clinical audiometer with a TDH-50P headphone, following the British Society of Audiology recommended procedure ${ }^{16-18}$. The audiometer was maintained and calibrated by the CAM services at the Royal National Throat, Nose \& Ear Hospital (RNTNE) to ANSI 3.6, ISO 389. The audiometer was checked subjectively according to the recommended procedure before the start of each clinic. Testing was carried out in a soundproof room in the Neuro-Otology clinic. The test was performed by following the BSA recommended procedures. $\mathrm{Pa}$ tients were asked to press the switch button or raise their fingers as soon as they heard the tone, and keep pressing the button until they no longer heard the tone. Tested frequencies were started at $1000 \mathrm{~Hz}$, followed by $2000 \mathrm{~Hz}, 4000 \mathrm{~Hz}, 8000 \mathrm{~Hz}, 500 \mathrm{~Hz}$, and $250 \mathrm{~Hz}$, and retest was done at $1000 \mathrm{~Hz}$, for the first ear only. Then, the opposite ear was tested following the same order. In a 3-second duration, tone was presented at a level that was expected to be clearly audible to the patients, which was commonly about 30 $\mathrm{dB}$ above the estimated threshold. Patient responses were checked if it was correct and indicated the entire duration. If there was no response, we raised the volume levels by $20 \mathrm{~dB}$-intervals until a response was obtained, and turned them down by $10 \mathrm{~dB}$ intervals until it dropped to $5 \mathrm{~dB}$ or until the patient no longer responded. Tones were presented up to 4 times to decide whether at least 2 satisfactory responses were received. If this level was heard, it was the threshold for that tone, if not, we raised the levels by $5 \mathrm{~dB}$ and repeat the same steps until 2 out of 4 presentations were satisfactory.

\section{Admittance Measures (Tympanometry)}

Impedance audiometry was carried out using GSI 33 Impedance Bridge, calibrated by CAM services at the Royal National Throat, Nose \& Ear Hospital to ANSI 3.6 (1969), ISO 389 (1975) standards. Screening tympanometry was carried out in all patients, according to the recommended procedures ${ }^{19}$. Patients were 
given a brief description of the test procedure. They were instructed to sit in a comfortable position and to avoid any unnecessary movement, speaking or swallowing after the probe was fitted into the ear canal. A clean tip of a suitable size and shape was selected for each patient by pulling the pinna upward and backward while inserting the probe with rotatory movement to obtain an airtight seal, and by applying an excessive pressure of $200 \mathrm{daPa}$ for a few seconds to ensure that the pressure was maintained. A routine measurement was done by selecting the probe frequency of $226 \mathrm{~Hz}$, with a slow rate of changes in pressure (50 daPa or less).

\section{Vestibular testing}

Rotational Test (Impulsive Rotation)

Rotational test was carried out using an ENG chair, GOULD TA 240S (GOULD Instrument systems, Inc) ISO9001-1987. On the test day, otoscopy and tympanometry were carried out to check the condition of the external ear canal and the tympanic membrane for each patient. Patients were given a detailed description of the test and the possible side effects while being seated on a rotational chair. Surgical sprit and cotton were used to clean the patient's forehead and outer canthi of the eyes with cautions. Three disposable silver-silver chloride electrodes were used. The ground electrode was placed on the center of the forehead, bitemporal electrodes were placed on outer canthi for conjugated horizontal eye movement recording. Electrode Jelly was applied through the hole in the center of the electrode using a syringe and a sterile blunt needle. The skin was gently abraded with the blunt needle to achieve impedance between 2 and $4 \mathrm{Ohms}$. The electrodes were then connected to the chair. ENG chair was connected to an integral light bar to adjust the patient head position relatively to the light bar and to perform eye calibration. Two calibration points were placed symmetrically on the horizontal plane in front of the patient for this purpose. After calibration, the impulse rotational test was carried out at $60^{\circ} / \mathrm{sec}$ in darkness. The biggest amplitude response for the right- and the left-sided nystagmus in the first 5 wave forms of the tracing after the start and stop of the stimulus was selected by manually measuring individual beats. The average for rightand left-sided nystagmus was calculated separately. Nystagmus was measured manually by drawing a line through the slow phase of a beat of nystagmus, then the line was extended above and below the limits of this beat so that it reached the margins of the paper.
After that, we counted $10 \mathrm{~mm}$ horizontally, as the paper speed was normally $10 \mathrm{~mm} / \mathrm{sec}$ and the slow component velocity (SCV) was specified in degrees/sec as a unit of measurement. Then a right-angled triangle was created by connecting the $10 \mathrm{~mm}$ time mark to the diagonal line running through the nystagmus beat. The numbers of millimeters that constituted this vertical line up to the intersection were the number of a degree per second for that nystagmus beat. The gain was calculated for each side by the following method: Gain = Maximum SCV/Stimulus (head/chair) Velocity

Comparing the responses from one side with the other symmetry was assessed by using the maximum SCV as follows:

Symmetry = VmR-VmL/VmR+VmL X 100; Where $\mathrm{Vm}$ is maximum SCV.

\section{Caloric Test}

Caloric test was carried out using the AQUAMETER water caloric stimulator by HORT MANN, GMBH, using Nicole 1992 by Nystar Computer program (Electronystagmography Testing \& Analysis). Patients were given a detailed description of the test and the possible side-effects before starting the test. They were advised to stop any medications, vestibular sedation and alcohol for up to 48 hours before testing. Electrodes were placed on patients as described in the previous method section. The patient was asked to lie supine on a couch with the head elevated at 30 degrees and was instructed to keep his gaze fixed at a small red indicator light on the ceiling in front of him and to do arithmetic through the test. The right ear was first irrigated with warm water $\left(44^{\circ} \mathrm{C}\right)$ from the caloric test machine for 40 seconds while ENG was recorded. This was followed by irrigation of the left ear with warm water after a short interval. Similarly, the ears were irrigated with cold $\left(30^{\circ} \mathrm{C}\right)$ water in the same order. A five minutes interval was given between each irrigation. The eyes were examined for nystagmus from the start of each irrigation with patients' gaze fixed on the target light on the ceiling, at first in light, and followed by darkness.

\section{Statistical Analysis}

Z-test was used to compare the current caloric and rotational test results with the data available from the literature and the normative data available in the Neuro-otology clinic. The subjects' audiograms were examined, and the subjects were divided into three groups including sensorineural hearing loss, conductive hearing loss, and mixed hearing loss. In a sensorineural hearing loss, the audiogram of the patients 
was below $20 \mathrm{~dB}$ hearing loss at any frequency or combination of frequencies. This would include flat and low-frequency losses and there was no air-bone gap of greater than $10 \mathrm{~dB}$ across the frequency range $0.5-4$ $\mathrm{KHz}$. In a conductive hearing loss, the air conduction threshold was below $20 \mathrm{~dB}$ hearing loss at any frequency in the frequency range $0.25-4 \mathrm{KHz}$. There would be an air-bone gap of greater than $10 \mathrm{~dB}$ hearing loss at least at one frequency in the range 0.5-4 $\mathrm{KHz}$, at which was a hearing loss. In a mixed hearing loss, the audiogram was below $20 \mathrm{~dB}$ hearing loss in both air conduction and bone conduction at any frequency in the frequency range $0.25-4 \mathrm{KHz}$. There would be an air-bone gap of greater than $10 \mathrm{~dB}$ hearing loss.

\section{RESULTS}

\section{Descriptive statistics of current study}

This study included 16 patients of 14 females and 2 males. Thirteen of the patients had radiologically confirmed otosclerosis (12 Females and 1 male). Three patients ( 2 females and 1 male) did not have CT confirmation of otosclerosis and therefore, were excluded from the study, although they had audiometric features of otosclerosis. The remaining 13 patient data were analyzed with regard to caloric, rotational chair testing, and questionnaire. Two patients were unable to do the caloric test and one other patient was unable to complete the test due to dizziness. Nine patients had a mixed hearing impairment at least on one side, 8 with bilateral mixed hearing loss and 1 with sensorineural hearing loss on the opposite side. Four patients had a bilateral sensorineural hearing loss.

Only 11 patients completed the questionnaire. Nine patients reported symptoms of dizziness. Additionally, they reported some forms of balance problems, including spinning in the head, a spinning of the surroundings, and light-headedness. Eight patients reported some precipitating factors that induced their dizziness. Five patients reported some associated aural symptoms such as hearing, tinnitus or both. Most of the patients reported the episodes of dizziness for a few months or for many years. The frequency of dizziness episode ranged from once a day to $1-2$ times a year.

One patient had a canal paresis (CP) at 35\% (normal ranges for $\mathrm{CP}$ is $0-20 \%$ ). This patient also had a symptom of dizziness. None of the patients had any significant directional preponderance (DP) (normal range for DP is $0-28 \%)$. The above patient with significant CP (35\%) did not show any rotational asymmetry on impulsive rotation.
Eleven patients had participated in a rotational chair test. Only one patient had a significant asymmetry to the right at $25.30 \%$ (normal range is $<20 \%$ ). However, this patient's caloric test did not show any significant CP or DP. Two patients (18\%) of the radiologically confirmed otosclerosis had an abnormal balance test, including both caloric and rotational tests.

\section{Comparison of current data with the avail- able data in the literature}

\section{Caloric test data}

One patient (12\%) in the current study had a significant CP. One out of 8 had an abnormal caloric test $=12 \%(p)$. Our result was compared with the literature $^{20}$, which reported that $60 \%$ of patients (total 191) had abnormal responses to bithermal caloric stimulation.

Cody \& Baker $60 \%(\Pi)$

$\mathrm{Z}=\mathrm{p}-\Pi / \sqrt{ } \mathrm{p}^{*}(100-\mathrm{p}) / \mathrm{n}$

$=$ number of patients in the study $=8$

$=12-60 / \sqrt{ } 12^{*}(100-12) / 8$

$=-48 / \sqrt{ } 12^{\star}(88) / 8$

$=-48 / \sqrt{ } 132$

$=-48 / 11.48$

$=4.1, \mathrm{p}<0.001$

Based on the normal distribution function table Probability $=p<0.001$ (2 tailed test), this result was translated into a significant level.

\section{Vestibular symptoms (questionnaire)}

Nine out of 11 patients in the current study had vestibular symptoms $(81.81 \%(p))$. We compared our result with the literature ${ }^{20}$. A study done by Cody and Baker ${ }^{20}$ tested 191 patients with both vestibular and auditory symptoms of otosclerosis. They found that about $42.5 \%$ of the patients had vestibular symptoms in their group.

Cody\& Baker $42.5 \%$ (П)

$\mathrm{Z}=\mathrm{p}-\Pi / \sqrt{ } \mathrm{p}^{\star}(100-\mathrm{p}) / \mathrm{n}$

$=$ number of patients in the study $=11$

$=81.81-42.5 / \sqrt{ } 81.81^{\star}(100-81.81) / 11$

$=39.31 / \sqrt{ } 81.81^{\star}(18.19) / 11$

$=39.31 / \sqrt{ } 1488.123 / 11$

$=39.31 / \sqrt{ } 135.228$

$=39.31 / 11.63$

$=3.4 \mathrm{p}<0.001$

Based on the normal distribution function table Probability $=p<0.001$ ( 2 tailed test), this result was translated into a significant level.

Therefore, in the current study, data about both caloric testing and vestibular symptoms were statically significant compared to the available data in the literature. 


\section{DISCUSSION}

Otosclerosis is the most frequent cause for those cases of progressive deafness, which may result in severe handicap. Vestibular disturbances in otosclerosis may arise from otosclerotic vascular changes of the abnormal spongy bone formation or from biochemical changes in the inner ear fluids. George Shambaugh, Jr., ${ }^{21}$ has observed that the inner ear damage in otosclerosis commonly occurs at the beginning of the disease, he was the first to describe a case of otosclerosis presenting at first with pure perspective deafness and only showing signs of stapedial ankylosis years later. Cody and Baker ${ }^{20}$ conducted a study in which they tested 191 patients with both vestibular and auditory symptoms of otosclerosis for three and a half years (between mid -1973 and December 1976). They found that about $60 \%$ of their patients had abnormal responses to bithermal caloric stimulation. A similar prevalence was reported by Virolanen ${ }^{22}$, who also found directional preponderance or positional nystagmus in one third to one half of the patients he studied. He found nystagmus in $41.17 \%$ of the patients on ENG. In the same study done by Cody and Baker ${ }^{20}$, they found that the incidence of vestibular symptoms increased as the relative and the absolute amount of sensorineural hearing loss increased. Not only did the incidence of the vestibular symptoms increase in the amount of sensorineural hearing loss, but also did the degree of severity of the vestibular symptoms.

In the current study, $12 \%(\mathrm{n}=1)$ of patients had an abnormal caloric test and $18.18 \%(n=2)$ had abnormal ENG findings, including both caloric and rotational tests. We did not attempt to compare our impulse rotation parameter, i.e. symmetry with the literature, as we were unable to find similar data. Our questionnaire analysis confirmed that $81.81 \%(\mathrm{n}=11)$ of our patients had some forms of dizziness. This is in contrast with Cody and Baker ${ }^{20}$ study, which reported a figure of $42.5 \%$ vestibular symptoms and $60 \% \mathrm{ab}$ normal response to caloric stimulation. This may be because our patients were a small group that possibly did not include those with significant vestibular test abnormalities.

The current study had many potential limitations. The number of patients tested in this study was small. The restrictions imposed by the ethics committee in carrying out the caloric test in normal individuals precluded control subjects for this study. The manual evaluation of maximum SCV used to assess gain and symmetry in impulse rotation test was known to be inaccurate and it also introduced an element of observer bias.

\section{CONCLUSIONS}

The current study suggests a high prevalence of balance symptoms $(81.81 \%, \mathrm{n}=9)$ in patients with radiological otosclerosis, although only a small proportion $(18.18 \%, \mathrm{n}=2)$ showed abnormal vestibular function on caloric and impulse rotation testing. However, due to a small number of samples in the current study, further studies on the larger sample are warranted to validate these findings.

\section{ABBREVIATIONS}

BSA: British Society of Audiology

CP: canal paresis

DP: directional preponderance

ENG: Electronystagmography

p: prevalence

PTA: Pure tone Audiometry

SCV: Slow component velocity

TM: Tympanic membrane

\section{COMPETING INTERESTS}

None of the authors reported any conflict interest related to this study.

\section{AUTHORS' CONTRIBUTIONS}

Rania Abdulfattah Sharaf: the corresponding author, participated in the study design, participated in the data collection, drafted the manuscript, and finalized the manuscript.

Rudrapathy Palaniappan: participated in the study design, helped with the ethics application and revised the manuscript critically.

\section{REFERENCES}

1. Cureoglu S, Baylan MY, Paparella MM. Cochlear otosclerosis. Current Opinion in Otolaryngology \{\&amp; $\}$ amp; Head \{\&amp;\}amp; Neck Surgery. 2010;18(5):357-62. 20693902. Available from: 10.1097/MOO.0b013e32833d11d9.

2. de Vilhena D, Gambôa I, Duarte D, Lopes G. Vestibular Disorders after Stapedial Surgery in Patients with Otosclerosis. International Journal of Otolaryngology. 2016;2016:6830648. 26904127. Available from: 10.1155/2016/6830648.

3. Parnes S, Black FO, Wall C, O'Leary DP, Feltyberger E. Vestibular system abnormalities in otosclerotic subjects. Otolaryngology. 1978;86(1):98-106. 114928. Available from: 10.1177/ 019459987808600122.

4. Redfors YD, Gröndahl HG, Hellgren J, Lindfors N, Nilsson I, Möller C. Otosclerosis: anatomy and pathology in the temporal bone assessed by multi-slice and cone-beam CT. Otology \{\&amp;\}amp; Neurotology. 2012;33(6):922-7. 22771999. Available from: 10.1097/MAO.0b013e318259b38c.

5. Faccioli N, Barillari M, Guariglia S, Zivelonghi E, Rizzotti $A_{\text {, }}$ Cerini $R$, et al. Radiation dose saving through the use of cone-beam CT in hearing-impaired patients. La Radiologia Medica. 2009;114(8):1308-18. 19789956. Available from: 10.1007/s11547-009-0462-y. 
6. Kanzara T, Virk JS. Diagnostic performance of high resolution computed tomography in otosclerosis. World Journal of Clinical Cases. 2017;5(7):286-91. 28798924. Available from: 10.12998/wjcc.v5.i7.286.

7. Quesnel AM, Ishai R, McKenna MJ. Otosclerosis: Temporal Bone Pathology. Otolaryngologic Clinics of North America. 2018;51(2):291-303. 29397947. Available from: 10.1016/j.otc. 2017.11.001.

8. Guild SR. Normal and Pathological Anatomy of the Petrous Pyramid. vol. CVl; 1935.

9. Nylen B. Histopathological investigations on the localization number, activity and extent of otosclerotic foci. The Journal of Laryngology and Otology. 1949;63(6):321-7. 18149650. Available from: 10.1017/S0022215100046533.

10. Cawthorne T. Otosclerosis. J Laryngol Otol. 1955;69(7):43756.

11. Virolainen E. Vestibular disturbances in clinical otosclerosis. Acta Oto-Laryngologica. 1972;74(sup306):5-34.

12. Panda NK, Saha AK, Gupta AK, Mann SB. Evaluation of vestibular functions in otosclerosis before and after small fenestra stapedotomy. Indian Journal of Otolaryngology and Head and Neck Surgery. 2001;53(1):23-7. 23119746. Available from: 10.1007/BF02910974.

13. Yetişer S. Bilateral Vestibulopathy Due to Severe Cochlear Otosclerosis: A Well-Known Condition Without Any Favorable Solution. Turk Arch Otorhinolaryngol. 2018;56(3):174-6. 30319876. Available from: 10.5152/tao.2018.3347.

14. Meurman $\mathrm{OH}$, Aantaa $\mathrm{E}$, Virolainen $\mathrm{E}$. Vestibular disturbances in clinical otosclerosis. Archives of Otolaryngology (Chicago, III). 1969;90(6):756-8. Available from: 10.1001/archotol.1969. 00770030758018 .
15. Morales-Garcia C. Cochleo-vestibular involvement in otosclerosis. Acta Oto-Laryngologica. 1972;73(6):484-92. 4537974 Available from: 10.3109/00016487209138969.

16. Moore DR, Rosen S, Bamiou DE, Campbell NG, Sirimanna T. Evolving concepts of developmental auditory processing disorder (APD): a British Society of Audiology APD special interest group 'white paper'. International Journal of Audiology. 2013;52(1):3-13. 23039930. Available from: 10.3109/ 14992027.2012 .723143$.

17. of Audiology BS. Pure tone air and bone conduction threshold audiometry with and without masking and determination of uncomfortable loudness levels. Reading, UK: British Society of Audiology; 2004

18. of Audiology BS. Recommended procedure for pure-tone bone-conduction audiometry without masking using a manually operated instrument. vol. 19. British Society of Audiology; 1985.

19. of Audiology BS. Recommended procedure for tympanometry. vol. 26. Bathgate EH47 7AQ: British Society of Audiology 1992.

20. Cody DT, Baker HL. Otosclerosis: vestibular symptoms and sensorineural hearing loss. The Annals of Otology, Rhinology, and Laryngology. 1978;87(6 Pt 1):778-96. 310648. Available from: 10.1177/000348947808700605.

21. Shambaugh GE. Endocrine aspects of Meniere's disease. The Laryngoscope. 1959;69(8):1027-32. 14445422. Available from: 10.1288/00005537-195908000-00004.

22. Terence C. Otosclerosis: The Dalby Memorial Lecture The Journal of Laryngology and Otology. 1955;69:437-56. 13242969. Available from: 10.1017/S0022215100050933. 